\title{
A Case of Accidental Formic Acid Poisoning
}

\author{
Sushil Kiran Kunder ${ }^{1}$, A. Avinash ${ }^{1}$, Sharath Madhyastha ${ }^{2 *}$, Raviraj Acharya ${ }^{2}$, Vinaya Gopalaswamy ${ }^{2}$, Girisha Balaraju ${ }^{3}$, \\ Navin Patil ${ }^{1}$, Amod Tilak ${ }^{1}$ \\ ${ }^{1}$ Dept. of Pharmacology, Kasturba Medical College, Manipal University, Manipal 576104, India. \\ ${ }^{2}$ Dept. of Medicine, Kasturba Medical College, Manipal University, Manipal 576104, India. \\ ${ }^{3}$ Dept. of Gastroenterology, Kasturba Medical College, Manipal University, Manipal 576104, India.
}

\begin{tabular}{l} 
ARTICLE INFO \\
\hline Article history: \\
Received on: $18 / 05 / 2016$ \\
Revised on: 14/06/2016 \\
Accepted on: $20 / 07 / 2016$ \\
Available online: $30 / 08 / 2016$ \\
\hline Key words: \\
Rubber, Corrosive, \\
Gastrointestinal bleed, \\
Caustic
\end{tabular}

\section{INTRODUCTION}

In the coastal regions of southern India, especially among the people working in rubber plantations, suicidal or accidental consumption of formic acid is a commonly encountered problem. The purpose of using formic acid is to coagulate rubber (More et al., 2014).

Therefore it is commercially available. Being corrosive and pungent, it is rarely consumed accidently. It is mostly used as a suicidal modality. It has been however, rarely used with homicidal intent too (Rajan et al., 1985). Formic acid is also used as a stain remover and descaling agent (Jefferys and Wiseman, 1980). The authors describe the clinical outcome of an elderly lady who had accidentally consumed formic acid.

\footnotetext{
* Corresponding Author

Sharath Madhyastha, Assistant Professor, Dept. of Medicine, Kasturba Medical College, Manipal University, Manipal 576104, India. Email:dr.sharathymc@gmail.com
}

\section{CASE HISTORY}

A 75-year-old lady (written informed consent obtained for publication), a known hypertensive, was admitted to Kasturba hospital with alleged accidental consumption of around $50 \mathrm{ml}$ of $85 \%$ diluted formic acid (a dilution of 1 litre formic acid in 100 litres of water). She had consumed the diluted poison thinking the bottle contained plain potable water. The presenting complaint was recurrent vomiting. There was no chest pain, shortness of breath, fever or abdominal pain. The patient had a history of upper gastrointestinal bleed about a year ago, which was diagnosed as non-steroidal anti-inflammatory drug-induced duodenal ulcer. In addition, she was on oral amitriptyline for depression since one year. On examination in the casualty, the patient was conscious and well oriented. Her vital signs were normal. Her systemic examination revealed bilateral $1 \mathrm{~mm}$-sized pupils (miosis was present). Higher motor functions, power and reflexes were normal. Routine blood investigations revealed elevated total leucocyte count $(15,000$ cells/cu.mm.) with neutrophilia (86.5\%). 
Serum electrolytes, liver function tests, renal function tests and urine examination were well within normal limits. Upper GI endoscopy showed Zargar grade 3 erosions in the lower oesophagus (as shown in figures 1 and 2).

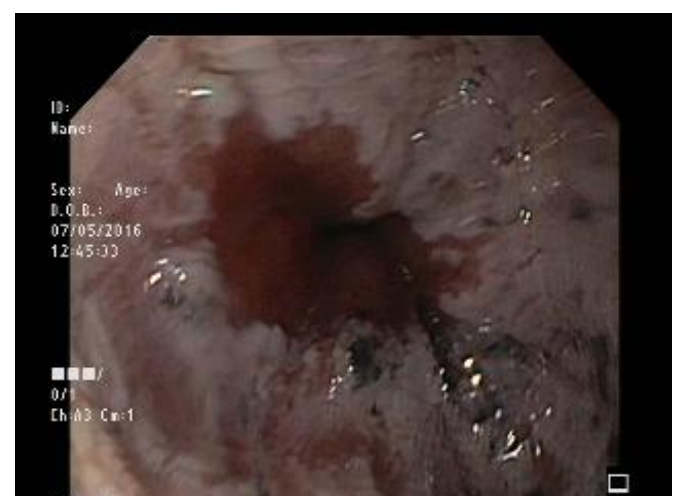

Fig. 1: Upper GI endoscopy showing oesophageal ulcerations and erosions.

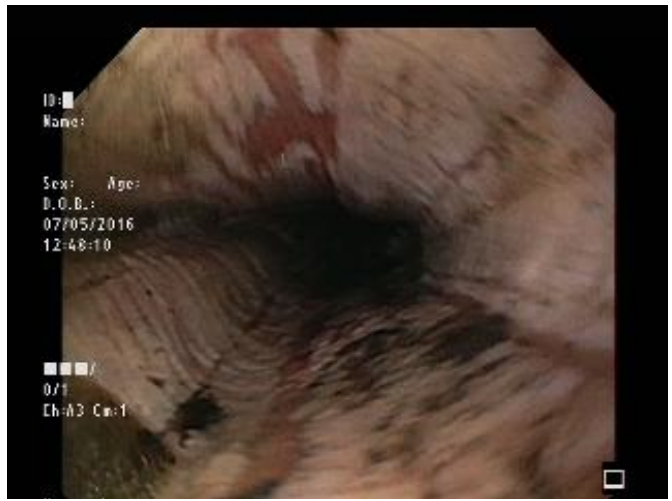

Fig. 2: Upper GI endoscopy showing oesophageal ulcerations and erosions.

The patient was started on intravenous fluids, intravenous pantoprazole $40 \mathrm{mg} 12^{\text {th }}$ hourly, intravenous ondansetron $4 \mathrm{mg} 8^{\text {th }}$ hourly, intravenous ceftriaxone $2 \mathrm{~g} 24^{\text {th }}$ hourly, oral sucralfate 15 $\mathrm{ml} 6^{\text {th }}$ hourly, oral quetiapine $12.5 \mathrm{mg}$ at bedtime and oral paracetomol $650 \mathrm{mg} 8^{\text {th }}$ hourly. The patient's antihypertensive medications were continued as before (oralamlodipine $5 \mathrm{mg}$ once daily and oral olmesartan $20 \mathrm{mg}$ once daily). The patient was started on Ryle's tube feeding to prevent esophageal stricture formation and advised to review for a follow-up endoscopic evaluation after 6 weeks (to reassess the status and to check for the need for esophageal dilatation). Six days following admission, the patient's total leucocyte count had normalized. The patient was discharged following improvement.

\section{DISCUSSION}

A case series reported as early as 1985 showcased 53 cases of suicidal consumption of formic acid in South India. Majority of these patients presented with gastrointestinal complaints including burns, ulcerations, abdominal pain, retching and vomiting (Rajan et al., 1985). A case series of 3 fatal formic acid poisoned patients also showed a predominance of gastrointestinal symptoms, like vomiting and haemetemesis
(Naik et al., 1980). Similarly, in the present case, gastrointestinal symptoms and signs overruled all other manifestations. Most of the patients from these two case series had accompanying leucocytosis, as evidenced in the present scenario (Rajan et al., 1985; Naik et al., 1980). Another case series that documented 45 cases of formic acid poisoning stated that only 2 of these patients had "accidently" consumed the poison, elucidating the fact this modality is more of a suicidal approach, owing to the fact that this is a highly pungent and corrosive agent (Jefferys and Wiseman, 1980). Ulcerations from the oral cavity to the duodenum were visualized either endoscopically or on the autopsy table (Rajan et al., 1985; Naik et al., 1980). In our case, the patient already had a history of duodenal ulceration, secondary to the use of NSAIDs. During the present admission, grade 3 erosions were found. Anuria and renal complications were also seen in several patients in these case reports found in literature (Rajan et al., 1985; Naik et al., 1980). However, this was not the case in our patient. Probably, the amount of formic acid consumed was comparatively less, or the patient might have vomited out the contents of the stomach before significant absorption had occurred. All the patients in the case series mentioned herewith were treated with non-specific symptomatic management, which comprised intravenous fluids, pain medication and sedation, whenever required (Rajan et al., 1985). A similar protocol was followed in our patient as well.

\section{CONCLUSION}

Formic acid remains a highly toxic compound that is consumed voluntarily or accidently, especially in the rubber industry population. While the most common presentation includes vomiting, haemetemesis and gastrointestinal ulceration, pneumonitis and renal failure may also intervene. There is no antidote or specific treatment for such cases, making it a difficult condition to diagnose and manage. Hence, medical care providers should always exclude this diagnosis, especially when managing a case of unknown poisoning. Further, protective measures should be undertaken to avoid accidental consumption of formic acid and other industrial poisons.

\section{REFERENCES}

Jefferys DB, Wiseman HM. Formic acid poisoning. Postgrad Med J, 1980;56:761-2.

More DK, Vora M, Wills V. Acute formic acid poisoning in a rubber plantation worker. Indian J Occup Environ Med 2014;18(1):29-31.

Naik RB, Stephens WP, Wilson DJ, Walker A, Lee HA. Ingestion of formic acid-containing agents - report of three fatal cases. Postgrad Med J, 1980;56:451-6.

Rajan N, Rahim R, Kumar SK. Formic acid poisoning with suicidal intent: a report of 53 cases. Postgrad Med J, 1985;61:35-6.

How to cite this article:

Kunder SK, Avinash A, Madhyastha S, Acharya R, Gopalaswamy V, Balaraju G, Patil N, Tilak A. A Case of Accidental Formic Acid Poisoning. J App Pharm Sci, 2016; 6 (08): 172-173. 